\title{
Market opportunities for small energy harvesters
}

\author{
Alessandra Cuneo ${ }^{1,}$, Stefano Barberis ${ }^{1}$, Alberto Traverso ${ }^{2}$, and Paolo Silvestri ${ }^{2}$ \\ ${ }^{1}$ RINA Consulting S.p.A., via San Nazaro 19, 16145 Genoa, Italy \\ ${ }^{2}$ Thermochemical Power Group, University of Genoa, Via Montallegro 1, 16145 Genoa, Italy
}

\begin{abstract}
There are several small energy sources that can be exploited to provide useful energy: small temperature differences, mechanical vibrations, flow variations, latent exhausts are just some examples. The recovery of such common and small energy sources, usually wasted, for example with the conversion into useful amounts of electrical energy, is called energy harvesting. Energy harvesting allows low-power embedded devices to be powered from naturally-occurring or unwanted environmental energy (e.g. pressure or temperature difference). The main aim in the last years of researches in such field, was the increasing of the efficiency of such components, with a higher power output and a smaller size. At present, a wide range of systems incorporating energy harvesters are now available commercially, all of them specific to certain types of energy source. Energy harvesting from dissipation processes such as fluid lamination is a challenge for many different applications. In addition, control valves to dissipate overpressures are common usage of many plants and systems. This paper surveys the market opportunities of such harvesting systems, considering the trade-offs affecting their efficiency, their applicability, and ease of deployment. Particular attention will be devoted to small energy harvesters than can exploit small expansions, such as from lamination valves or to systems that can feed mini sensors from small pressure drops, promising compactness, efficiency and cost effectiveness.
\end{abstract}

\section{Introduction}

Energy harvesting is the process in which a small amount of energy, that would otherwise be lost such as heat, light, sound, vibration, wind, magnetic force or movement, is transformed into an electric current and stored for later usage [1]. It is well-known for its applications in solar cells and electro dynamo but it knows numerous new innovative uses thanks to recent digital trends, especially Internet of Things (IoT). Energy harvesting is also a great tool to address the issue of climate change and global warming because it reuses ambient energy which is otherwise wasted. The most common energy sources used for energy harvesting are mechanical, thermal energy and solar radiation [2]. Figure 1 gives an overview of which technologies are used for energy harvester. There is an increasing attention on energy harvesting in the last years, driven both by a higher energy/environmental responsibility (which drives energy harvesting application in large scale installation like waste heat valorisation, fluid expansion valorization, etc.) and by an increasing need of power for sensors in the modern IoT environment (which drives technological and market development for technologies like piezo, thermoelectric, micro$\mathrm{PV}$, etc.).

\footnotetext{
* Corresponding author: alessandra.cuneo@rina.org
} 


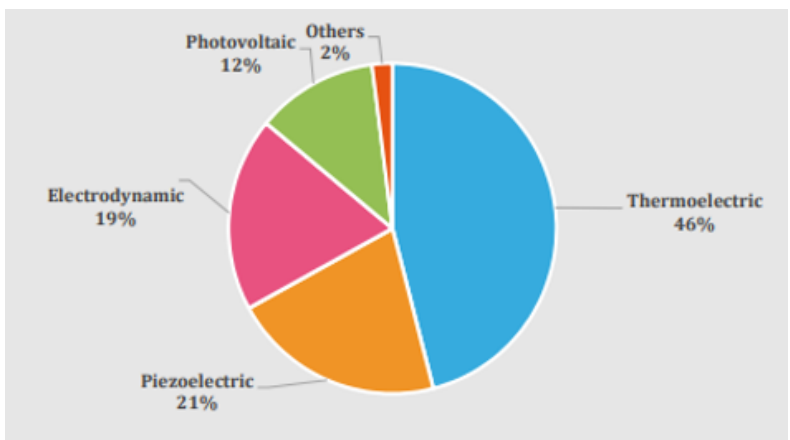

Fig. 1. Global energy harvesting market by technology [2]

\section{Current Energy Harvesting Market Scenario}

The global energy harvesting market was valued at $1.2 \mathrm{~B} €$ in 2014 and has been anticipated to reach $2.6 \mathrm{~B} €$ by 2020 according to several studies conducted by the Energy Harvesting Journal or by Winter Green Research [3]. Green energy trend is boosting the market as plenty of renewable energy sources as well as the growing demand for building and home automation and the development of Big Data and IoT technologies and their need to replace batteries in monitoring/sensoring devices.

Looking at the different applications where the energy harvester technologies could be applied, commercial and residential are the most important application areas (Fig.2). Smart houses and home automation as energy harvesting is enabling the development of technologies that would have not been economically possible without it.

The second most important application area is defence (unmanned drones and reduction of battery weight in soldier backpacks). The energy harvesting market in the industrial segment forecasts to increase in the next years also leveraging on the increasing sensitivity to industrial energy efficiency and reliability in the whole industrial process.

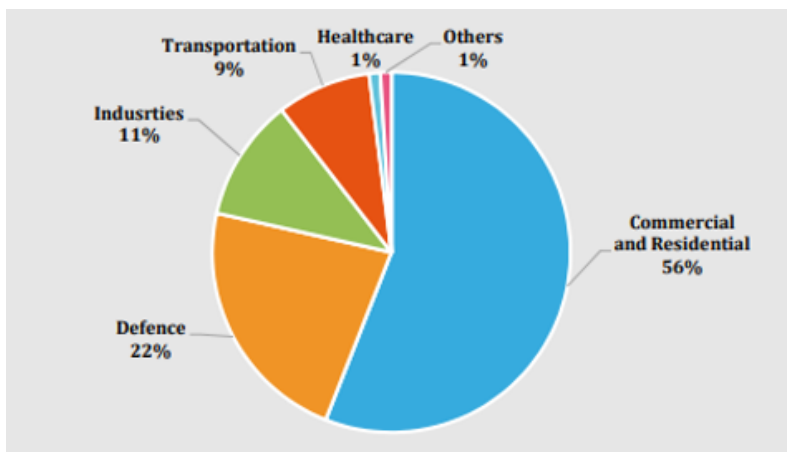

Fig. 2 Global energy harvesting market 2014-2019 [2]

Application of energy harvesting remains limited due to its small conversion efficiency (around 5\%) and its low energy storage capacity. Therefore, the utilisation of energy harvesting remains limited to low-power-consuming devices. The high entry costs and the unstable output of electricity through energy harvesting can be seen as further barriers to energy harvesting market exploitation. The key driver behind the energy harvesting market growth is the replacement of battery-powered sensors by autonomous energy harvesting wireless sensors. Harvesters are replacing batteries because they lower maintenance fee and are more environmentally friendly. They are even more relevant in remote or hostile 
environments where it is complex and costly to change batteries (i.e. single battery change for an offshore/desert area oil or gas system costs over 1M€) [4]. Unfortunately, in this kind of sector, as well as in the industrial one, innovation is not always able to penetrate if compared with state-of-the-art and business-as-usual technologies/applications. The power generated by the different energy harvesting technologies could vary between few microwatts up to some $\mathrm{kW}$ [5]. The generated energy can cover multiple orders of magnitude.

\section{Expansion fluid energy harvesters}

In order to attract investments in the field of energy harvesting in industrial application and to pursue waste heat/waste energy valorisation processes that are currently promoted in different industrial sectors, micro-turbines and blade less turbines could be turnkey technology considering their low capital costs, their easy integration in processes and the possibility to work with different types of fluid (air, refrigerants etc.), even with small pressure drops. Furthermore, their implementation could be considered both in microscale specialized applications (i.e. sensors along a gas pipeline powered by a micro-turbine where gas from local venting evolves in the machine $-5-50 \mathrm{~W}$ ), domestic small scale ones (i.e. replacing the expansion valve of a fridge/conditioning unit reducing its electric consumption - up to $150 \mathrm{~W}$ ) [6] and industrial ones (i.e. exploiting pressurized air vents or replacing expansion valve in large refrigerators - up to $10 \mathrm{~kW}$ ). One important aspect to be considered in this kind of turbomachinery is obviously how to guarantee a proper functioning and reliability of the machine in case of phase transition of the operating fluid and the related presence of liquid and gaseous phases. The proper coupling of the harvesters with generator and power electronics have to be duly considered, in order to minimize losses and costs in this part of energy production value chain considering, unfortunately, the already low conversion efficiency of such harvesters (3-10\%).

\section{Conclusion}

In this brief overview, market perspective, needs and barriers for energy harvesters are presented with a special focus on fluid expansion harvesters, which could effectively operate in different sectors and sizes. In order to guarantee reliable and cost-effective performance, further experimental activities have to be promoted (like those ones performed in H2020 PUMP-HEAT project by UNIGE [7]). This kind of R\&D investment seems promising considering the market growth of the energy harvesting sector.

\section{Acknoledgements}

This project has received funding from the European Union's Horizon 2020 research and innovation programme under Grant Agreement No 764706, PUMP-HEAT.

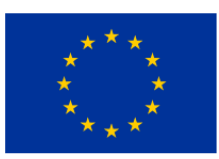

\section{References}

[1] A. Harb, «Energy harvesting: State-of-the-art,» Renewable Energy, Bd. 36, pp. 2641 2654, 2011.

[2] Energy harvesting to power the rise of the Internet of Things - EC - Digital Transformation Monitoring, July 2017

[3] Business Innovation Observatory, Energy harvesting, 2014.

[4] P. Harrop, R.Das, Energy Harvesting and Storage for Electronic Devices 2010-2020, 2010.

[5] P. Harrop, «Introduction to Energy Harvesting \& Off-Grid Renewable Energy,» in IDTechEx Show! Emerging Technologies Unleashed, Berlin, 2017. 
[6] A. Renuke, A. Vannoni, M. Pascenti, A. Traverso, Experimental and Numerical Investigation of Small Scale Tesla Turbines, GT2019-91352, ASME Turbo Expo 2019, Phoenix (AZ)

[7] https://www.pumpheat.eu/ (last access 02/05/2019) 